\title{
Application and Related Issues of Biotechnology in Insect Classification
}

\author{
Jiani Liu ${ }^{1, a}$, Yuyu Zhang ${ }^{2, b}$, Shengguang $\mathrm{Xu}^{3, \mathrm{c}}$, Zebin Chen ${ }^{4, d}$, Lei $\mathrm{Yu}^{5, \mathrm{e}_{*}}$ \\ 1,2,3,4 Kunming University \\ 1,2,3,4 Yunnan Urban Agricultural Engineering \& Technological Research Center \\ ${ }^{1,2,3,4}$ Key Laboratory of Special Biological Resource Development and Utilization of Universities in \\ Yunnan Province, Kunming, 650214, China
}

Keywords: Application, Related Issues, Biotechnology, Insect Classification

\begin{abstract}
This paper summarizes the research progress of biotechnology in insect taxonomy in recent ten years and introduces the technology of biotechnology such as RFLP, RAPD, PCR, nucleic acid sequence analysis, probe hybridization and its application in insect taxonomy. It pointed out that insect taxonomy, especially systematic theory is a comprehensive and highly disciplinary subject, and the comprehensive application of various biotechnologies is the research direction of insect molecular systems.
\end{abstract}

\section{Introduction}

At present, there are more than 1 million species of insects, accounting for $2 / 3$ of the known animals, but about $90 \%$ of the world's insects are unknown species. With the progress of science and technology and the interdisciplinary penetration of various disciplines, insect taxonomy has been developed for more than 200 years especially the application of modern biotechnology has accelerated the development of insect taxonomy. For a long time, insect classification is mainly based on external morphological characteristics. Because the external morphological features are more intuitive and easy to master, and according to the morphological characteristics of insects as a classification based on the classification unit can be a good reflection of the classification of species status. However, in the small classification unit, such as family, family, species is not easy to determine the classification of species status, and then to the population, ecological type is more difficult to determine the classification status. Since the 1970s, gas chromatography or temperament-mass spectrometry, nucleic acid sequence analysis, random amplified polymorphic DNA (RAPD), restriction fragment length polymorphism (RFLP) Molecular hybridization, single strand conformation polymorphism (SSCP) and double strand conformation polymorphism (DSCP) technology, which greatly promoted the development of insect taxonomy.

\section{The Insect Molecular Ecology Research Methods}

Isoenzyme (Allele) Electrophoresis. Isozyme refers to enzymes of different molecular forms with the same substrate specificity, with tissue, development and species specificity. The basic principle is based on the difference in charge properties, by protein electrophoresis or chromatographic techniques and specialized staining reactions show different forms of isoenzymes, which identify different genotypes.

Isozyme markers can reflect the genetic variation of the organism from the protein level. By analyzing the allele frequency, the percentage of polymorphic loci and the distribution and distribution of alleles in the population, we can know the genetic structure of the species. In addition, the study of polymorphic loci can explore the differentiation of the population, provide the genetic basis for the division of the subspecies, or explore and establish the interspecific boundaries. In combination with the ecological conditions of the population for isozyme analysis and DNA analysis, you can also understand the genetic basis of the biocompatible environment. In recent years, esterase isoenzymes have also been widely used to identify the genetic variation of pest resistance.

Isozymes as a genetic marker also has its limitations, there is evidence that the study results 
underestimated the variation of insect population, only $30 \%$ of the genetic diversity can be detected, so the enzyme electrophoresis may underestimate genetic variation Level. In addition, the isoenzyme is the product of gene expression, genotype different nature will lead to different isozymes and thus susceptible to the development stage of the enzyme, environmental conditions. But because of its economic, convenient, easy to operate, is still the most widely used genetic marker technology.

RAPD. PAPD technology is the 20th century, 90 years in the PCR technology, based on a single artificial synthesis of random primers (usually 10bp) on the genomic DNA amplification and development of a DNA polymorphism molecular marker technology, called the random expansion Increased polymorphism. RAPD technology can provide information of genetic variation quickly and easily. It is widely used in insect population genetics research, including the degree of genetic variation of population and genetic differentiation among populations, identification and identification of natural enemy insects, identification of pest biotypes, Genetic markers to identify kinship. RAPD techniques also play an important role in detecting variability associated with pest resistance and analyzing the genetic structure of insect populations and tracing trajectories of insect migration.

RAPD technique has the characteristics of fast, simple and sensitive in the study of entomology, which is very beneficial to the study of small insects. In addition, since RAPD does not need to know the sequence of templates, and the primer sequence is not genetically specific, it has a special advantage in genetic analysis of species with little genetic background. Of course, RAPD also has a reaction that is too sensitive to detect heterozygotes and is highly susceptible to exogenous DNA (such as contaminating microorganisms, in vivo symbiosis and parasite DNA).

RFLP. RFLP (restriction fragment length polymorphism) is a new technology developed in the 1980s to study biological polymorphism. The principle of restriction endonuclease (RE) digestion of different individual genomic DNA, after electrophoresis separation performance restriction fragment length difference, this difference can be carried out by specific probe hybridization, and then autoradiography Show up [6]. Commonly used probes are derived from random clones or cD-NA clones of single or low copy genomic DNA.

RELP markers were Mendelian inheritance, not environmental impact, can distinguish between homozygous genotypes and heterozygous genotypes, the results are more reliable and stable, particularly suitable for species identification or intraspecific population genetic evolution studies to build gene linkage map, in the It is also a powerful tool for the analysis and evaluation of gene flow among populations and in pedigree and genetic relationships. However, RELP analysis can only detect mutations in the endonuclease recognition site, provide limited information, and probe preparation is also cumbersome. At present, the target molecule selected by RFLP is mtDNA, which can be used to detect the difference of sequence or population among groups, group phylogenetic study, individual identification, hybridization and so on.

DNAfp (DNA fingerprint). 1985 Jiffreys and other human myoglobin sites obtained by the 33bp core sequence as a probe to study the individual, the resulting DNA map varies from individual to individual, like a human fingerprint, this individual and species of DNA The map says DNA fingerprints. This method is to detect the sequence of cells in the sequence of repeated sequence units. DNAfp can be found in DNA fingerprints of both parents, in addition to occasional mutations, so DNAfp is especially suitable for studies of parental discrimination, individual identification, population and interspecific relationships.

SSCP and DSCP. SSCP (Single Strand Conformation Polymorphism) and DSCP (Double Strand Conformation Polymorphism) are simple and rapid methods to detect DNA mutations in recent years. DSCP is developed on the basis of SSCP and DNA molecules without denaturation can be directly electrophoresis detection. By SS-CP and DSCP analysis, the molecular variation of DNA-specific fragments can be detected. It is mainly used for the study of insect population genetic and evolutionary biology.

This method has the advantages of rapid, simple and economic as RAPD and RELP, but is more stable and repeatable than RAPD. It is a quick way to realize molecular marker. The mutated bands 
are cloned and sequenced. The disadvantage of this method is that some mutations do not cause DNA bending and do not change the conformation of the molecule and thus cannot be detected. While DNA bending can be reasonably determined from known sequences, many DNA sequences can produce the same electrophoretic band type.

Probe Hybridization Technology. The molecular hybridization technology of nucleic acid is one of the most widely used techniques in molecular biology. It is not only an important part of molecular cloning technology, but also plays an important role in gene expression, regulation and phylogenetic relationship effect. Nucleic acid molecules that have a certain homology of DNA single-stranded molecules or DNA single-stranded molecules and RNA molecules, and its complementary segments can be renatured after denaturing denaturation conditions to form double-stranded DNA molecules or DNA / RNA hetero-double chains The molecular hybridization techniques of nucleic acids have different forms and methods, including Southern molecular hybridization, Northern molecular hybridization and in situ hybridization. The genetic polymorphism, DNA homology and mRNA difference of insects were detected by nucleic acid molecular hybridization technique. The identification of insects and geographical populations was the molecular biotechnology commonly used in the current insect system research.

\section{The Application of Modern Biotechnology in Insect Ecology}

Population Genetic Structure Analysis. Population genetic structure refers to the total genetic variation within a species and its distribution pattern among groups is a kind of the most basic genetic information. The study of genetic structure of insect population can not only estimate the genetic polymorphism of population, the range and mode of gene flow among populations, but also infer the structure of ancestral population and establish the relationship between phylogenetic relationships among populations. Methods such as isometric enzyme electrophoresis, RAPD, RELP, SSCP and DSCP are commonly used to study the genetic structure of insect populations. There are many studies in this field, mainly in the genetic variation of esterase isoenzymes, nDNA, mtDNA and other genetic populations in different geographical populations.

The Identification of Biological Type. Biological type (or host host races) is usually considered to be the same species within the genetic type. The study of biology is helpful in understanding its formation, differentiation mechanism, and the relationship between insects and hosts. The identification of the general biological type is mainly based on the inoculation reaction of the host or other biological habits to identify, not only time-consuming and difficult to use for practical identification, the application of molecular biology technology to make the identification of insect biology more accurate and reliable.

The Identification of Pest Resistance. The detection of drug resistance is a research field developed in recent years due to the large number of pesticides used to produce insect pests. In the past, we have studied from the aspects of physiology and rarely penetrate into the molecular level. In recent years, esterase isoenzymes have been widely used as a genetic variation to identify pest resistance. RAPD has also played an important role in detecting mutations related to pest resistance. In the absence of drug-resistant genes, In the case of knowledge, the genetic variation of resistance to populations can be identified by DNA polymorphism markers linked to the resistance gene.

Identification and Use of Natural Enemies. In the biological control, the correct identification of natural enemies is a key factor in the success of prevention and control, which is not only in the selection, the release of natural enemies early, but also in the future management, assessment of control effect is also very important. However, in the identification of natural enemies often encounter some obstacles, such as the kind of complex with the same kind of native species, and most natural enemies and their host individual small, these problems often difficult to identify the target natural enemies, molecular marker technology in entomology In the application of a good solution to these problems. Roehrdanz and other RAPD techniques identified the different geographical origin of the North American ladybug, for the correct introduction of natural enemies and control aphid harm played a decisive role, also pointed out that RAPD technology can be used to distinguish the relationship between the natural enemies. These aspects of the study in the use of 
natural enemies control pests have great economic significance, will be the identification of insects, the introduction and lay a solid theoretical foundation.

Prophase Recognition. Clonal identification refers to the ability of social insects to identify relatives and non-relatives, which is considered a necessary condition for the social origin and evolution of insects. In addition, the pro-recognition of insects in the individual communication, collaboration and reproduction and other social life plays an important role. In this field, the study mainly focused on the identification of the phylogenetic relationship of the genus ophiopogon japonicus.

The Study of the Insect Diapause Mechanism. Diapause is a kind of genetically controlled growth and development stagnation in insects. It is an important ecological mechanism for regulating the life history of herbivorous insects and synchronizing with the host plant phenology and avoiding adverse environmental conditions. Studies have shown that photoperiod is the main factor in the induction, maintenance and termination of diapause and the temperature, humidity and foodstuff play an auxiliary and concomitant role. With the rapid development of biochemistry and modern molecular biology and the increasing use in the study of entomology, the research on insect diapause has made a breakthrough.

Study the Relationship between Insects and Host Plants. The close relationship between phytophagous insects and their hosts has an important effect on the genetic structure of insect populations. One case is the discontinuity of the host's spatial distribution, or the sub-population is specialized in the feeding of a host; the other is that the restriction of gene flow between the host-related subgroups contributes to the adaptability of the species and the evolution of resource development strategies. At present, Isoenzyme markers are useful tools for studying gene flow patterns and the degree of genetic differentiation among species and hosts within insects.

\section{Conclusion}

The development of modern biotechnology has accelerated the study of insect ecology in the microscopic field. Its simple and quick research methods have greatly accelerated the process of life science research, so that some of the life phenomena that cannot be explained by traditional methods and theories can be obtained from its genetic mechanism explanation. The application of molecular biology techniques in insect ecology is still in its infancy and has been applied to all aspects of insect ecology research. It will play an important role in insect ecology research.

\section{References}

[1] Jia Xinzhang, Li Jingyuan. Journal of Northwest Agricultural University, Vol. 6 (2014) No 53, p.25-26

[2] Peng Sue, Wang Yunhui, Wang Qunyong. Insect Knowledge, Vol. 12 (2015) No 27, p.74-76

[3] Qian Xiyuan, Hou XuSiem. Journal of Insect Taxonomy, Vol. 30 (2014) No 19, p.144-145

[4] Wang Kuailiang. Journal of Beijing Forestry University, Vol. 29 (2008) No 27, p.21-23

[5] Zhang Gongxu, Sun Jing. Animal Science and Animal Science, Vol. 8 (2013) No 27, p.57-60 